Supplementary Information for the paper

\title{
Discrete Binuclear Co(III) bis-Dioximates with Wheel-and-axle Topology as Building Blocks to Afford Porous Supramolecular Metal-organic Frameworks
}

Eduard Coropceanu, ${ }^{\mathrm{a}}$ Andrei Rija, ${ }^{\mathrm{a}}$ Vasile Lozan, ${ }^{\mathrm{a}}$ Ion Bulhac, ${ }^{\mathrm{a}}$ Gheorghe Duca, ${ }^{\mathrm{a}}$ Victor Ch. Kravtsov, ${ }^{\text {b,* }}$ and Paulina Bourosh ${ }^{\mathrm{b}, *}$

${ }^{a}$ Institute of Chemistry, Academy of Sciences of Moldova, Academiei str. 3, MD2028 Chisinau, Republic of Moldova;

${ }^{\mathrm{b}}$ Institute of Applied Physics, Academy of Sciences of Moldova, Academiei str. 5, MD2028

Chisinau, Republic of Moldova

Table 1S. Selected Bond Distances $(\AA)$ and Angles $\left(^{\circ}\right)$ for $\mathbf{1}-\mathbf{4}$

\begin{tabular}{|c|c|c|c|c|c|}
\hline \multirow[t]{2}{*}{ Compound } & \multirow[t]{2}{*}{1} & \multicolumn{2}{|l|}{2} & \multirow[t]{2}{*}{3} & \multirow[t]{2}{*}{4} \\
\hline & & A & B & & \\
\hline $\mathrm{Co}(1)-\mathrm{N}(11)$ & $1.896(3)$ & $1.92(1)$ & $1.839(11)$ & $1.896(2)$ & $1.897(2)$ \\
\hline $\mathrm{Co}(1)-\mathrm{N}(12)$ & $1.894(3)$ & $1.88(1)$ & $1.860(11)$ & $1.899(2)$ & - \\
\hline $\mathrm{Co}(1)-\mathrm{N}(21)$ & $1.895(3)$ & $1.88(1)$ & $1.862(11)$ & $1.904(2)$ & - \\
\hline $\mathrm{Co}(1)-\mathrm{N}(22)$ & $1.905(4)$ & $1.89(1)$ & $1.883(10)$ & $1.902(2)$ & $1.888(2)$ \\
\hline $\mathrm{Co}(1)-\mathrm{N}(31)$ & $1.960(3)$ & $1.914(9)$ & $1.978(8)$ & $1.950(2)$ & $1.960(3)$ \\
\hline $\mathrm{Co}(1)-\mathrm{Cl}(1)$ & $2.232(1)$ & $2.245(4)$ & $2.234(3)$ & $2.2419(6)$ & $2.223(1)$ \\
\hline \multirow[t]{2}{*}{ Compound } & \multirow[t]{2}{*}{1} & 2 & & \multirow[t]{2}{*}{3} & \multirow[t]{2}{*}{4} \\
\hline & & A & $\mathrm{B}$ & & \\
\hline $\mathrm{N}(11)-\mathrm{Co}(1)-\mathrm{N}(12)$ & $81.1(2)$ & $80.4(5)$ & $80.6(6)$ & $81.48(9)$ & - \\
\hline $\mathrm{N}(11)-\mathrm{Co}(1)-\mathrm{N}(11) \# 1$ & - & - & - & - & $81.08(13)$ \\
\hline $\mathrm{N}(11)-\mathrm{Co}(1)-\mathrm{N}(21)$ & $179.4(2)$ & $178.3(5)$ & $178.4(7)$ & $178.75(9)$ & \\
\hline $\mathrm{N}(11)-\mathrm{Co}(1)-\mathrm{N}(22) \# 1$ & - & - & - & - & $178.27(10)$ \\
\hline $\mathrm{N}(11)-\mathrm{Co}(1)-\mathrm{N}(22)$ & $98.9(2)$ & $99.5(7)$ & $99.1(6)$ & $99.51(10)$ & $99.14(9)$ \\
\hline $\mathrm{N}(11)-\mathrm{Co}(1)-\mathrm{N}(31)$ & $89.7(1)$ & $91.6(4)$ & $87.9(4)$ & $89.36(6)$ & $91.45(9)$ \\
\hline $\mathrm{N}(11)-\mathrm{Co}(1) \mathrm{Cl}(1)$ & $90.5(1)$ & $89.9(3)$ & $90.8(3)$ & $88.88(6)$ & $89.34(7)$ \\
\hline $\mathrm{N}(12)-\mathrm{Co}(1)-\mathrm{N}(21)$ & $98.5(2)$ & $98.6(6)$ & $100.9(6)$ & $97.93(9)$ & - \\
\hline $\mathrm{N}(12)-\mathrm{Co}(1)-\mathrm{N}(22)$ & $178.9(1)$ & $178.5(5)$ & $176.7(4)$ & $178.93(9)$ & - \\
\hline $\mathrm{N}(12)-\mathrm{Co}(1)-\mathrm{N}(31)$ & $90.3(1)$ & $91.2(4)$ & $91.2(4)$ & $88.95(8)$ & \\
\hline $\mathrm{N}(12)-\mathrm{Co}(1)-\mathrm{Cl}(1)$ & $89.8(1)$ & $88.9(3)$ & $87.5(3)$ & $90.12(6)$ & - \\
\hline $\mathrm{N}(21)-\mathrm{Co}(1)-\mathrm{N}(22)$ & $81.5(2)$ & $81.4(7)$ & $79.3(6)$ & $81.06(9)$ & - \\
\hline $\mathrm{N}(21)-\mathrm{Co}(1)-\mathrm{N}(31)$ & $89.9(1)$ & $89.8(4)$ & $91.8(4)$ & $91.73(8)$ & - \\
\hline $\mathrm{N}(21)-\mathrm{Co}(1)-\mathrm{Cl}(1)$ & $89.90(9)$ & $88.7(4)$ & $89.5(3)$ & $90.02(6)$ & - \\
\hline $\mathrm{N}(22)-\mathrm{Co}(1)-\mathrm{N}(22) \# 1$ & - & - & - & - & $80.59(13)$ \\
\hline $\mathrm{N}(22)-\mathrm{Co}(1)-\mathrm{N}(31)$ & $90.8(1)$ & $90.3(4)$ & $92.1(4)$ & $91.45(9)$ & $90.26(9)$ \\
\hline $\mathrm{N}(22)-\mathrm{Co}(1)-\mathrm{Cl}(1)$ & $89.04(9)$ & $89.6(4)$ & $89.2(2)$ & $89.50(7)$ & $88.94(7)$ \\
\hline $\mathrm{N}(31)-\mathrm{Co}(1)-\mathrm{Cl}(1)$ & $179.8(1)$ & $178.5(3)$ & $178.3(2)$ & $178.11(6)$ & $178.95(9)$ \\
\hline
\end{tabular}

Symmetry transformations used to generate equivalent atoms:

$\# 1$ in $1-x+5 / 3,-y+1 / 3,-z+4 / 3$, \#1 in $2, \mathrm{~A}-x+1 / 2,-y+3 / 2, z$, \#1 in $2, \mathrm{~B}-x+1,-y+1,-z$, $\# 1$ in $3-x,-y+1,-z+1$, \#1 in $\mathbf{4} x,-y+1, z$

Table 2S. Hydrogen Bonds $(\AA)$ and Angles $\left(^{\circ}\right)$ for $\mathbf{1}-\mathbf{4}$.

\begin{tabular}{l|l|l|l|l}
\hline $\mathrm{D}-\mathrm{H}^{\cdots} \mathrm{A}$ & $\mathrm{d}\left(\mathrm{H}^{\cdots} \mathrm{A}\right)$ & $\mathrm{d}(\mathrm{D} \cdots \mathrm{A})$ & $(\mathrm{DHA})$ & $\begin{array}{l}\text { Symmetry transformation } \\
\text { for acceptor }\end{array}$ \\
\hline
\end{tabular}




\begin{tabular}{|c|c|c|c|c|}
\hline \multicolumn{5}{|l|}{1} \\
\hline $\mathrm{O}(12)-\mathrm{H} \cdots \mathrm{O}(21)$ & 1.70 & $2.490(4)$ & 161 & $x, y, z$ \\
\hline $\mathrm{O}(22)-\mathrm{H} \cdots \mathrm{O}(11)$ & 1.68 & $2.478(5)$ & 163 & $x, y, z$ \\
\hline $\mathrm{C}(13)-\mathrm{H} \cdots \mathrm{Cl}(1)$ & 2.75 & $3.616(5)$ & 151 & $-x+y+4 / 3,-x+2 / 3, z-1 / 3$ \\
\hline $\mathrm{C}(23)-\mathrm{H} \cdots \mathrm{O}(1 \mathrm{D})$ & 2.59 & $3.36(1)$ & 137 & $x, y, z$ \\
\hline $\mathrm{C}(34)-\mathrm{H} \cdots \mathrm{O}(21)$ & 2.59 & $3.493(5)$ & 165 & $x-y+1 / 3, x-1 / 3, z+5 / 3$ \\
\hline $\mathrm{C}(2 \mathrm{D})-\mathrm{H} \cdots \mathrm{O}(11)$ & 2.45 & $3.27(1)$ & 144 & $x, y, z-1$ \\
\hline $\mathrm{C}(2 \mathrm{D})-\mathrm{H} \cdots \mathrm{Cl}(1)$ & 2.72 & $3.55(1)$ & 145 & $x-y+1 / 3, x-1 / 3,-z+5 / 3$ \\
\hline \multicolumn{5}{|l|}{2} \\
\hline $\mathrm{O}(12 \mathrm{~A})-\mathrm{H} \cdots \mathrm{O}(21 \mathrm{~A})$ & 1.68 & $2.47(2)$ & 159 & $x, y, z$ \\
\hline $\mathrm{O}(22 \mathrm{~A})-\mathrm{H} \cdots \mathrm{O}(11 \mathrm{~A})$ & 1.67 & $2.48(2)$ & 168 & $x, y, z$ \\
\hline $\mathrm{O}(21 \mathrm{~B})-\mathrm{H} \cdots \mathrm{O}(12 \mathrm{~B})$ & 1.74 & $2.53(1)$ & 161 & $x, y, z$ \\
\hline $\mathrm{O}(22 \mathrm{~B})-\mathrm{H} \cdots \mathrm{O}(11 \mathrm{~B})$ & 1.80 & $2.56(1)$ & 155 & $x, y, z$ \\
\hline $\mathrm{O}(1 \mathrm{~W})-\mathrm{H}(2) \cdots \mathrm{O}(11 \mathrm{~B})$ & 2.08 & $2.93(2)$ & 178 & $-x+1 / 2,-y+3 / 2, z$ \\
\hline $\mathrm{C}(15 \mathrm{~A})-\mathrm{H} \cdots \mathrm{O}(21 \mathrm{~B})$ & 2.57 & $3.39(3)$ & 143 & $-x+1 / 2,-y+1 / 2, z$ \\
\hline $\mathrm{C}(16 \mathrm{~B})-\mathrm{H} \cdots \mathrm{Cl}(1)$ & 2.93 & $3.59(2)$ & 127 & $-x+1 / 2, y, z-1 / 2$ \\
\hline \multicolumn{5}{|l|}{3} \\
\hline $\mathrm{O}(12)-\mathrm{H} \cdots \mathrm{O}(21)$ & 1.68 & $2.472(3)$ & 163 & $x, y, z$ \\
\hline $\mathrm{O}(22)-\mathrm{H} \cdots \mathrm{O}(11)$ & 1.73 & $2.521(3)$ & 162 & $x, y, z$ \\
\hline $\mathrm{O}(1 \mathrm{~W})-\mathrm{H}(1) \cdots \mathrm{O}(11)$ & 1.90 & $2.779(7)$ & 165 & $x, y, z$ \\
\hline $\mathrm{O}(1 \mathrm{~W})-\mathrm{H}(2) \cdots \mathrm{O}(22)$ & 1.93 & $2.799(7)$ & 163 & $-x+1,-y, z+1$ \\
\hline $\mathrm{C}(24)-\mathrm{H} \cdots \mathrm{Cl}(1)$ & 2.86 & $3.640(3)$ & 139 & $x-1, y, z$ \\
\hline $\mathrm{C}(31)-\mathrm{H} \cdots \mathrm{O}(1 \mathrm{~W})$ & 2.41 & $3.192(7)$ & 142 & $x-1 / 2,-y+1 / 2, z+1 / 2$ \\
\hline $\mathrm{C}(32)-\mathrm{H} \cdots \mathrm{Cl}(1)$ & 2.74 & $3.550(3)$ & 146 & $-x+1 / 2, y+1 / 2,-z+3 / 2$ \\
\hline $\mathrm{C}(34)-\mathrm{H} \cdots \mathrm{Cl}(1)$ & 2.85 & $3.721(2)$ & 157 & $x-1 / 2,-y+1 / 2, z-1 / 2$ \\
\hline \multicolumn{5}{|l|}{4} \\
\hline $\mathrm{O}(22)-\mathrm{H} \cdots \mathrm{O}(11)$ & 1.70 & $2.492(3)$ & 162 & $x, y, z$ \\
\hline $\mathrm{O}(1 \mathrm{~W})-\mathrm{H}(1) \cdots \mathrm{Cl}(1)$ & 2.60 & $3.344(3)$ & 147 & $x, y, z$ \\
\hline $\mathrm{C}(31)-\mathrm{H} \cdots \mathrm{O}(22)$ & 2.38 & $3.162(3)$ & 142 & $-x+3 / 2,-y+3 / 2,-z+2$ \\
\hline $\mathrm{C}(42)-\mathrm{H} \cdots \mathrm{O}(1 \mathrm{~W})$ & 2.57 & $3.324(4)$ & 139 & $x+1 / 21, y-1 / 2, z$ \\
\hline
\end{tabular}

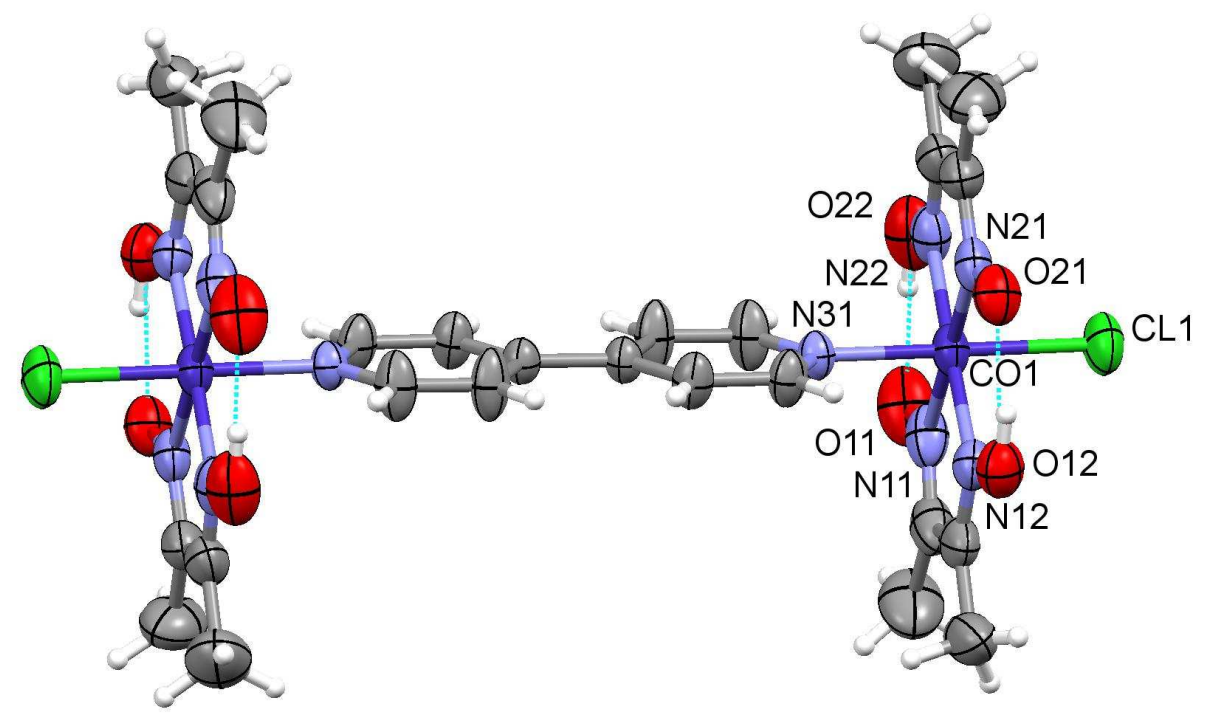



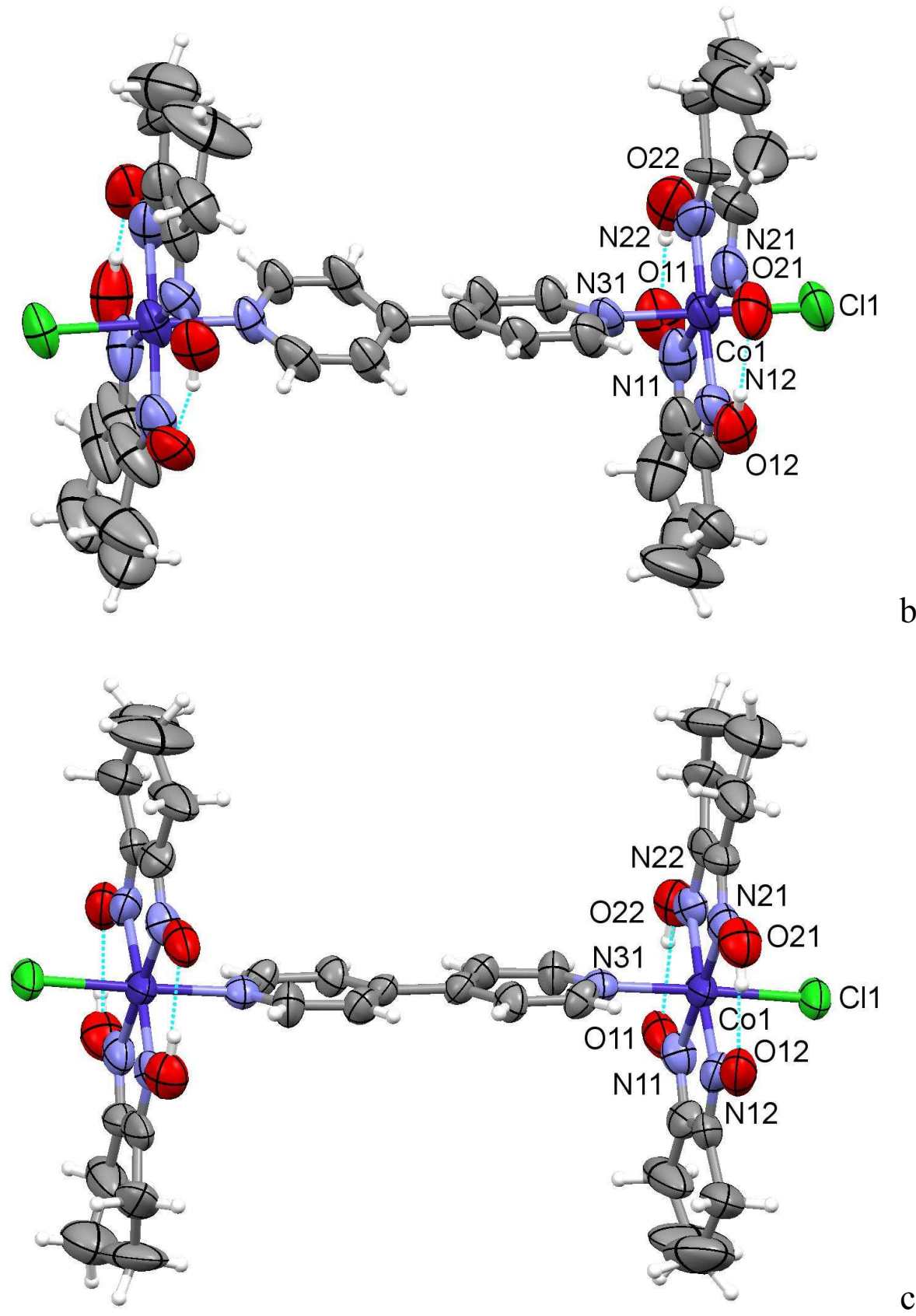

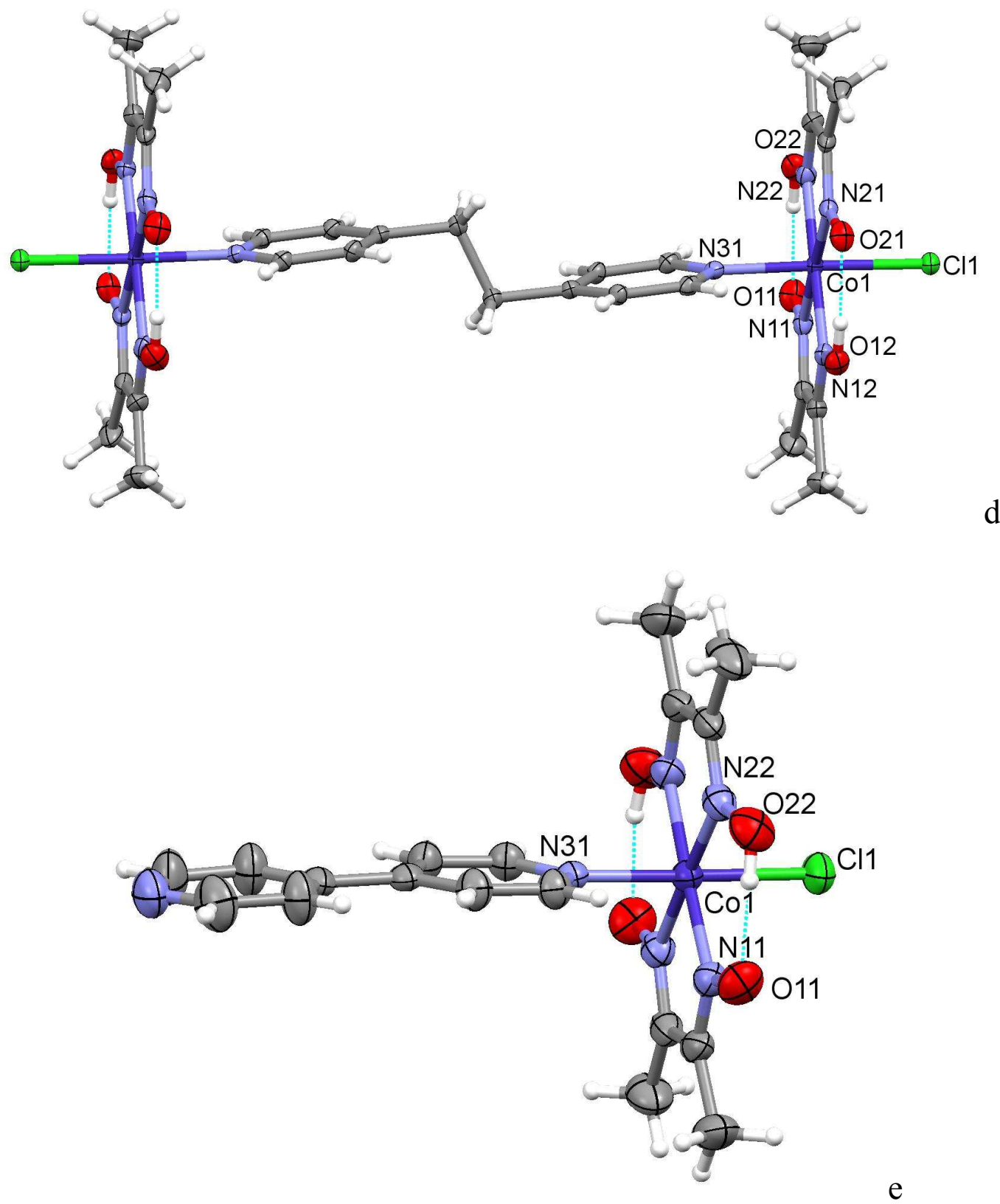

Fig. 1. ORTEP drawing for $\mathbf{1}-\mathbf{4}$ with a partial atomic numbering scheme (50\% thermal ellipsoids) and a shortest intramolecular $\mathrm{O}-\mathrm{H} \cdots \mathrm{O}$ distances shown by dotted lines: $\mathrm{a}-\mathbf{1}, \mathrm{b}$ and $\mathrm{c}$ $-\mathrm{A}$ and $\mathrm{B}$ complexes in $\mathbf{2}, \mathrm{d}-\mathbf{3}$, and $\mathrm{e}-\mathbf{4}$. 